Macedonian Pharmaceutical Bulletin, 66 (Suppl 1) 235 - 236 (2020)

Online ISSN 1857 - 8969

UDC: 606:577.216]:613.25

DOI: 10.33320/maced.pharm.bull.2020.66.03.117

Short communication

\title{
Nanostructured lipid carriers as drug delivery systems for miRNA
}

\author{
Amina Tucak ${ }^{1}$, Merima Sirbubalo ${ }^{1}$, Jasmina Hadžiabdić ${ }^{1}$, Ognjenka Rahić ${ }^{1}$, \\ Ivana Ruseska $^{2}$, Andreas Zimmer ${ }^{2} *$, Edina Vranić ${ }^{1}$ \\ ${ }^{1}$ University of Sarajevo, Faculty of Pharmacy, Department of Pharmaceutical Technology, Zmaja od Bosne 8, \\ 71000 Sarajevo, Bosnia \& Herzegovina \\ ${ }^{2}$ University of Graz, Institute of Pharmaceutical Sciences, Department of Pharmaceutical Technology and \\ Biopharmacy, 8010 Graz, Austria
}

\section{Introduction}

MicroRNAs (miRNAs) represent endogenous small RNAs that post-transcriptionally regulate gene expression and, thus they are involved in the onset and progression of various diseases and conditions (Bader et al., 2010) such as for overweight and obesity. Antiadipogenic miRNA-27a is a negative regulator in fat metabolism, which inhibits adipocyte differentiation through downregulation of adipogenic marker genes (e.g. PPAR $\gamma$ ) (Kim et al., 2010).

Reduced miRNA-27a levels are often associated with the development of obesity and, therefore, this miRNA might represent a promising candidate for miRNA mimic replacement therapy (Lin et al., 2009). However, the application of naked RNAs has shown low membrane permeability, cellular uptake, and rapid degradation in the circulation.

The present study aimed to develop a cationic, lipid-based nanoparticle system for targeting adipose tissue and delivering miRNA-27a. These systems are composed of positively charged nanostructured lipid carriers (cNLCs) and negatively charged miRNAs, which results in complex formation based on electrostatic interactions between these components.

\section{Materials and methods}

For the preparation of cNLCs, stearylamine (SA) (Sigma-Aldrich, Germany), Miglyol ${ }^{\circledR}$ 812, (Herba Chemosan Apotheker-AG, Austria), Precirol ${ }^{\circledR}$ ATO 5 (Gattefossè Deutschland $\mathrm{GmbH}$, Germany), Tween $^{\circledR} 80$ (Sigma-Aldrich, Germany), Pluronic ${ }^{\circledR}$ F68 (BASF, USA), and ultra-purified water, Milli$\mathrm{Q}^{\circledR}$ (Millipore S.A.S., France) were used. Doublestranded miRNA-27a with the sequence UUCACAGUGGCUAAGUUCCGC (Dharmacon, GE Healthcare, Austria) was purchased. Nuclease free water (VWR, Austria) was utilized to reduce the risk of nucleic acid degradation.

\section{Production of nanostructured lipid nanocarriers}

Stearylamine $\left(0.15 \%\right.$; w/w), Precirol ${ }^{\circledR}$ ATO 5 (4.365\%), and Miglyol ${ }^{\circledR} 812(0.485 \%)$ were melted at $70{ }^{\circ} \mathrm{C}$. The aqueous solution composed of Tween ${ }^{\circledR}$ $80(1 \%)$ and Pluronic ${ }^{\circledR}$ F68 (1\%) was simultaneously heated to the same temperature and added to the lipid phase. The coarse emulsion was obtained by mixing for $60 \mathrm{sec}$ at $8000 \mathrm{rpm}$ using the Ultra Turrax (IKA1-Werke GmbH \& Co., Germany) and then transferred into the high-pressure homogenizer

* andreas.zimmer@uni-graz.at 
(Panda 2K, NS1001L, GEA Niro Soavi, Germany) operating at 800 bar and $70{ }^{\circ} \mathrm{C}(5$ cycles $)$.

\section{Formation and characterization of miRNA-cNLC complexes}

The stock solution of miRNA-27a was diluted with RNase-free water to $1.3 \mu \mathrm{M}$ solutions. The working solutions of the cNLCs and miRNA-27a were intermixed using equal volumes and incubated at room temperature for $15 \mathrm{~min}$ and $5 \mathrm{~min}$ of sonication (Emmi-D100, EMAG Technologies, Germany) containing final miRNA concentration of $650 \mathrm{nM}$. To obtain samples suitable for the particle size $(100 \mathrm{nM})$ and zeta potential $(80 \mathrm{nM})$ measurements, complexes were diluted with RNasefree water. We investigated the influence on nanoparticle assembly and physicochemical properties of different mass ratios (miRNA27a: SA) ranging from 5:1 to 1:5.

Particle size, polydispersity index (PdI), and zeta potential (ZP) were determined by dynamic light scattering (DLS) and electrophoretic light scattering (ELS) with the Zetasizer Nano ZS (Malvern Instruments).

\section{Gel Electrophoresis}

To evaluate if there is any unbound miRNA in complexes left, E-Gel Power Snap Electrophoresis System with the E-GelTM Power Snap Camera (Thermo Fisher Scientific Inc, Austria) was used. Briefly, $20 \mu \mathrm{L}$ of miRNA27-a, and complexes miRNA-27a: SA (1:1 to 1:25) were loaded into the wells of the $4 \% \mathrm{E}^{-G e l}{ }^{\mathrm{TM}}$ agarose gel.

\section{Results and discussion}

The characterization of particle size by PCS revealed that the average particle size of the cNLC on the production date was $102.30 \pm 0.45 \mathrm{~nm}$, and the PdI of $0.166 \pm 0.011$ showed that formulation has unimodal distribution. Since the addition of SA increases the $\mathrm{pH}$ of formulations due to the presence of primary ammonium groups in its structure, $\mathrm{pH}$ was measured immediately after production $(\mathrm{pH}$ $8.89 \pm 0.07)$. The $\mathrm{pH}$ was thus, adjusted to around 7 to produce a full ionization of SA molecules at the interface of NLCs and, subsequently, the ZP was measured $(31.73 \pm 0.46 \mathrm{mV})$.
Complexation of cNLCs with miRNA-27a in ratios from $5: 1$ to $1: 5$ led to particle sizes ranging from $238.00 \pm 3.88 \mathrm{~nm}(1: 5)$ to $123.5 \pm 1.56 \mathrm{~nm}(5: 1)$. However, the highest particle size $(430.8 \pm 24.68 \mathrm{~nm})$ and PdI of $0.354 \pm 0.056 \mathrm{~nm}$ were observed in a complex that contained SA and miRNAs in equal mass ratio due to the low $\mathrm{ZP}(4.09 \pm 0.09 \mathrm{mV})$ that is related to the formation of aggregates of lipid phase. $\mathrm{ZP}$ measurements revealed a shift from a negative value $(-20.50 \pm 0.62 \mathrm{mV})$, in case of $5: 1$ ratio, to positive surface charges $(28.50 \pm 0.48 \mathrm{mV})$, in $1: 5$ ratio. This result suggests that cationic cNLCs nature enables electrostatic interactions with miRNAs to form self-assembled particles. Gel electrophoresis showed that above 1:5 mass ratio, there was no presence of unbounded miRNA, suggesting the successful cNLC-miR-27a complex formation.

\section{Conclusion}

The physicochemical investigations using DLS, ELS, and gel electrophoresis have shown the selfassembled nature of cNLC-miRNA-27a as complexes. Therefore, future studies are planned in an in vitro cell culture model to study the antiadipogenic effect of miRNA-27a on adipogenesis by applying our cNLC-based DDS for nucleic acids.

\section{Acknowledges}

Financial support from Scientific \& Technological Cooperation between Austria and B\&H - OeAD - Austrian Agency for International Cooperation in Education and Research (grant ref. BIH 09/2019), is acknowledged.

\section{References}

Bader, A.G., Brown, D., Winkler, M., 2010. The Promise of MicroRNA Replacement Therapy. Cancer Res. 70(18), 7027-7030.

Kim, S.Y., Kim, A.Y., Lee, H.W., Son, Y.H., Lee, G.Y., Lee, J.W., et al. 2010. miR-27a is a negative regulator of adipocyte differentiation via suppressing PPARgamma expression. Biochem. Biophys. Res. Commun. 392(3), 323-328.

Lin, Q., Gao, Z., Alarcon, R. M., Ye, J., Yun, Z., 2009. A Role of MiR-27 in the Regulation of Adipogenesis. FEBS J. 276(8), 2348-2358.

Maced. Pharm. Bull. 66 (Suppl 1) 235 - 236 (2020) 